\title{
Spinodal decomposition of Fe-Cu nanocrystals: Control of atomic-magnetic-moment and magnetic properties
}

\author{
P. Crespo \\ Instituto de Magnetismo Aplicado, Universidad Complutense, P.O. Box 155, Las Rozas 28230 Madrid, Spain
}

A. Hernando

Material Science Department, Cambridge University, Cambridge CB2 3QZ, United Kingdom

A. Garcia Escorial

Centro Nacional de Investigaciones Metalúrgicas, Avda G. del Amo, 8, 28040 Madrid, Spain

(Received 24 January 1994)

\begin{abstract}
Experimental results corresponding to the saturation magnetization and coercive field during the decomposition, upon annealing, of bcc and fcc $\mathrm{Fe}_{x} \mathrm{Cu}_{1-x}$, obtained by mechanical alloying are reported. The overall behavior points out that the decomposition takes place in two steps: (i) at low temperatures a decrease of the saturation magnetic moment as well as an anomalous thermal dependence of coercive field are observed, however, no phase transformation is detected, and (ii) for further annealing temperatures a new phase appears; the magnetization tends to increase and the coercive field abruptly increases. The analysis of the results leads us to conclude that the first step corresponds to a spinodal decomposition. Fluctuations in the local composition give rise to coexistence of adjacent regions with Curie temperature varying continuously in a range of $1000 \mathrm{~K}$ across distances of a few nanometers, thus allowing the tailoring of the magnetic nanostructures.
\end{abstract}

Some recent works have been focused on the preparation by mechanical alloying of $\mathrm{FeCu}$ alloys, ${ }^{1-3}$ which were produced earlier by vapor deposition. ${ }^{4}$ Less studies have been devoted to the change of the magnetic properties through the decomposition process. Nevertheless, a surprising drastic decrease of the magnetic moment per $\mathrm{Fe}$ atom during decomposition of the metastable ferromagnetic fcc $\mathrm{Fe}_{51} \mathrm{Cu}_{49}$ solid solution has been recently reported. ${ }^{5}$ This behavior cannot be explained by the simple decomposition of the system into the stable bcc Fe and $\mathrm{fcc} \mathrm{Cu}$ phases, and it was proposed that the separation may take place via a spinodal mechanism. ${ }^{6}$ Subsequent experimental studies on the same alloy have shown that during decomposition a broad range of different magnetic behavior is achieved, ranging from that of the magnetically soft as-milled sample to the hard magnetic behavior observed at intermediate stages. It was also indicated that the thermal dependence of the magnetization shows typical features of multiphase magnetic systems. ${ }^{7}$

The aim of this letter is (i) to show that spinodal decomposition ${ }^{8,9}$ takes place during the first steps of the separation process of $\mathrm{FeCu}$ alloys, (ii) to point out that this mechanism can produce drastic changes in the spontaneous magnetization, keeping constant the average composition, and (iii) to explain how that decomposition process allows the control of the coercivity and its thermal dependence through the control of the exchange correlation length.

$\mathrm{Fe}_{x} \mathrm{Cu}_{1-\mathrm{x}}$ alloys obtained by sputtering or by mechanical alloying are fcc for Fe content below 60 at. \% and bcc above 60 at. $\% .^{4,6}$ The experimental magnetic moment, measured at $10 \mathrm{~K}$, increases linearly with the Fe content $x$ and there is no noticeable discontinuity at $x=0.6 .^{10}$ The magnetic moment corresponding to either fcc phase in the range $0.6<x<1$ or bcc phase in the range $0<x<0.6$ remains unknown. However, the magnetic moment of $\mathrm{Fe}$ in the fcc phase should decrease from 1.35 Bohr magnetons at $x=0.6$ (Ref. 10) to zero at $x=1$ (fcc or $\gamma-\mathrm{Fe}$ ). It also seems reasonable to assume that the magnetic moment of the bcc phase vanishes as $x$ approaches 0 (bcc $\mathrm{Cu}$ ).

Spinodal fluctuations do not involve any crystalline phase transformation but a spatial modulation of the local composition, with wavelength of about $3.5 \mathrm{~nm}$, as has been recently observed by means of field ion microscopy in $\mathrm{CoCu}$ alloys obtained by melt spinning. ${ }^{8}$ Hence, if an $\mathrm{FeCu}$ alloy decomposes via spinodal mechanism, the magnetic moment as well as the Curie temperature would fluctuate according to the compositional modulation.

Metastable bcc $\mathrm{FeCu}, x=0.7$ and fcc $\mathrm{FeCu}, x=0.5$ and 0.3 solid solutions have been obtained by mechanical alloying in a Fritsch vibrating mill under a pressure of 1.5 atm of argon. The resulting powder was studied in asmilled state and after heating up to several temperatures to follow the decomposition of the metastable alloy into the stable phases. The heating was carried out in a differential scanning calorimeter at $20 \mathrm{~K} / \mathrm{min}$ under argon atmosphere. High-resolution $x$-ray-diffraction patterns were obtained at very low step velocity using $\mathrm{Cu}$ $K \alpha$ radiation. The composition and purity of the sample was checked by scanning electron microscopy, equipped with energy dispersion x-ray analysis, and gas analyzer. Nitrogen contamination was not detected. Thermogravimetric measurements showed that oxidation during an- 
nealing was smaller than $0.1 \%$. The thermal dependence of the magnetization has been determined by using a superconducting quantum interference device, Quantum Design MPMS. Grain size was estimated from the $\mathrm{Cu}$ (111) and $\mathrm{Fe}(110)$ peaks by using Voigt analysis. ${ }^{11}$

The x-ray-diffraction data of the samples as milled show a single phase structure. Upon heating at $723 \mathrm{~K}$ a new phase, bcc for samples $x=0.3$ and 0.5 and fcc for sample $x=0.7$, appears and grows with further thermal treatments (Fig. 1). The analysis of the $x$-ray-diffraction data leads to the grain size, plotted in Fig. 1 as a function of the heating temperature $T_{t}$. Figure 2 illustrates the magnetic moment, at $5 \mathrm{~K}$, under an applied field of $5 \mathrm{~T}$ corresponding to the three compositions in the as-milled state and after different thermal treatments. Figure 3 illustrates the influence of the heating on the coercive field. The thermal variation of coercivity for samples $x=0.3$ and 0.7 , as milled and after heating at different $T_{t}$, are also shown.

It turns out that prior to the appearance of the new phase some type of atomic rearrangements, which do not involve any transformation of crystalline structure, takes place. These rearrangements are associated with a decrease of the atomic magnetic moment observed in the low-temperature range of Fig. 2, and a change in the coercive field as well as in its thermal variation, as illustrated by Fig. 3. The phase transformation produces a

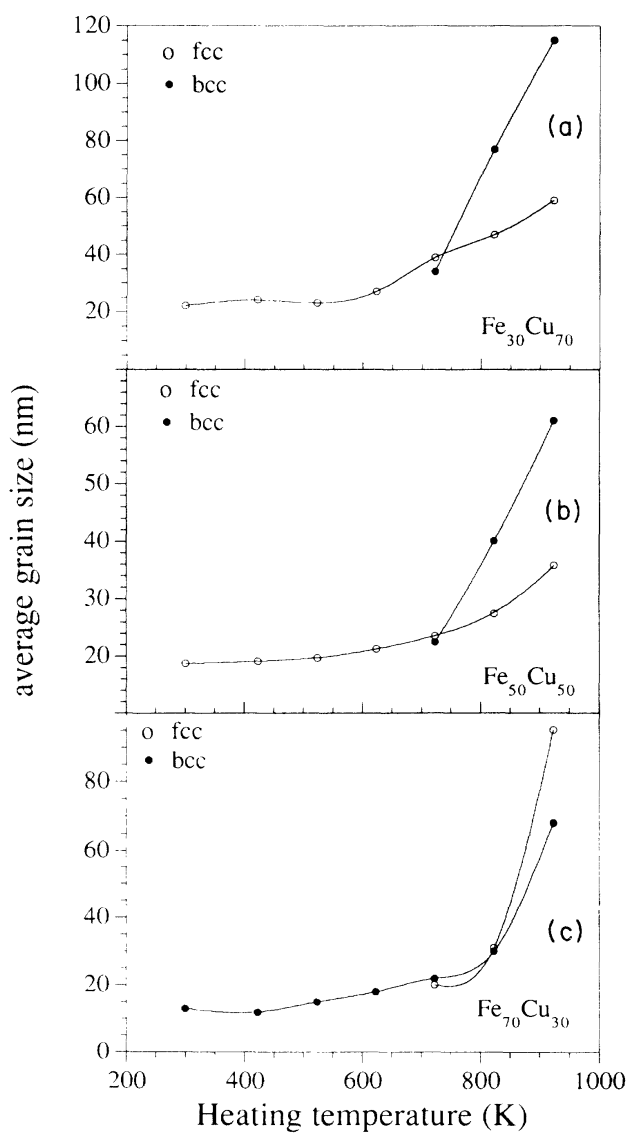

FIG. 1. Evolution of the grain size with the temperature of thermal treatment for the three alloys: (a) $x=0.3$, (b) $x=0.5$, (c) $x=0.7$. magnetic hardening. When the new phase is bcc the growth of its volume fraction [Figs. 1(a) and 1(b)] gives rise to an increase of the magnetic moment [Figs. 2(a) and 2(b)] and a slow decrease of the coercivity [Fig. 3(c)]. These two effects are not observed when the new phase is fcc [Figs. 1(c), 2(c), and 3(c)]. It is worth noting that the onset for the appearance of the new phase is detected through coercivity earlier than through $\mathrm{x}$-ray diffraction (Figs. 1 and 3).

The coercive field of samples consisting of a single magnetic phase varies with temperature as the inverse of the product of the saturation magnetization and the exchange correlation length. As the anisotropy decreases with temperature faster than the exchange interactions, the exchange correlation length increases with temperature. In general this increasing rate is faster than that of the magnetization decrease and thus the coercive field decreases with temperature. In heterogeneous samples consisting of a mixture of different magnetic phases the thermal dependence of the exchange correlation length shows anomalous behavior close to the Curie temperature of the magnetic phases. These anomalies are reflected in the thermal variation of coercivity. ${ }^{12,13}$ Annealed samples show a thermal dependence of coercivity typical of heterogeneous magnetic materials. The increase of coercivity with temperature exhibited by sample with $x=0.3$ after $T_{t}=723 \mathrm{~K}$ [Fig. 3(a)] may be understood as due to the shortening of the exchange correla-

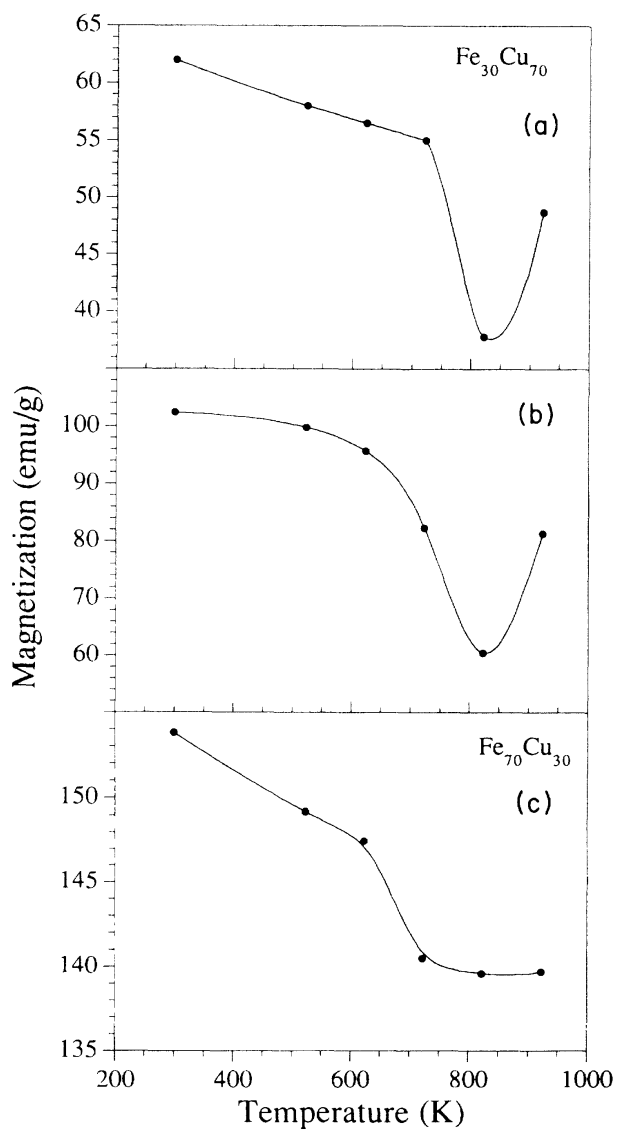

FIG. 2. Dependence of the magnetization, measured at $5 \mathrm{~K}$, on the temperature of thermal treatment: (a) $x=0.3$, (b) $x=0.5$, (c) $x=0.7$. 
tion length produced by the transformation from ferromagnetic to paramagnetic of a volume fraction of the material which lead to exchange isolation of some ferromagnetic regions. This effect is illustrated in a more fascinating way by the transformation observed in the bcc sample, $x=0.7$, after heating at $T_{t}=523 \mathrm{~K}$. In this case a transition from ferromagnetic to superparamagnetic behavior occurs at $150 \mathrm{~K}$ as illustrated in Fig. 3(b). The appearance of superparamagnetism was also checked by measuring the magnetization at different temperatures and then plotting the results as a function of the applied field reduced to the measuring temperature. Since for
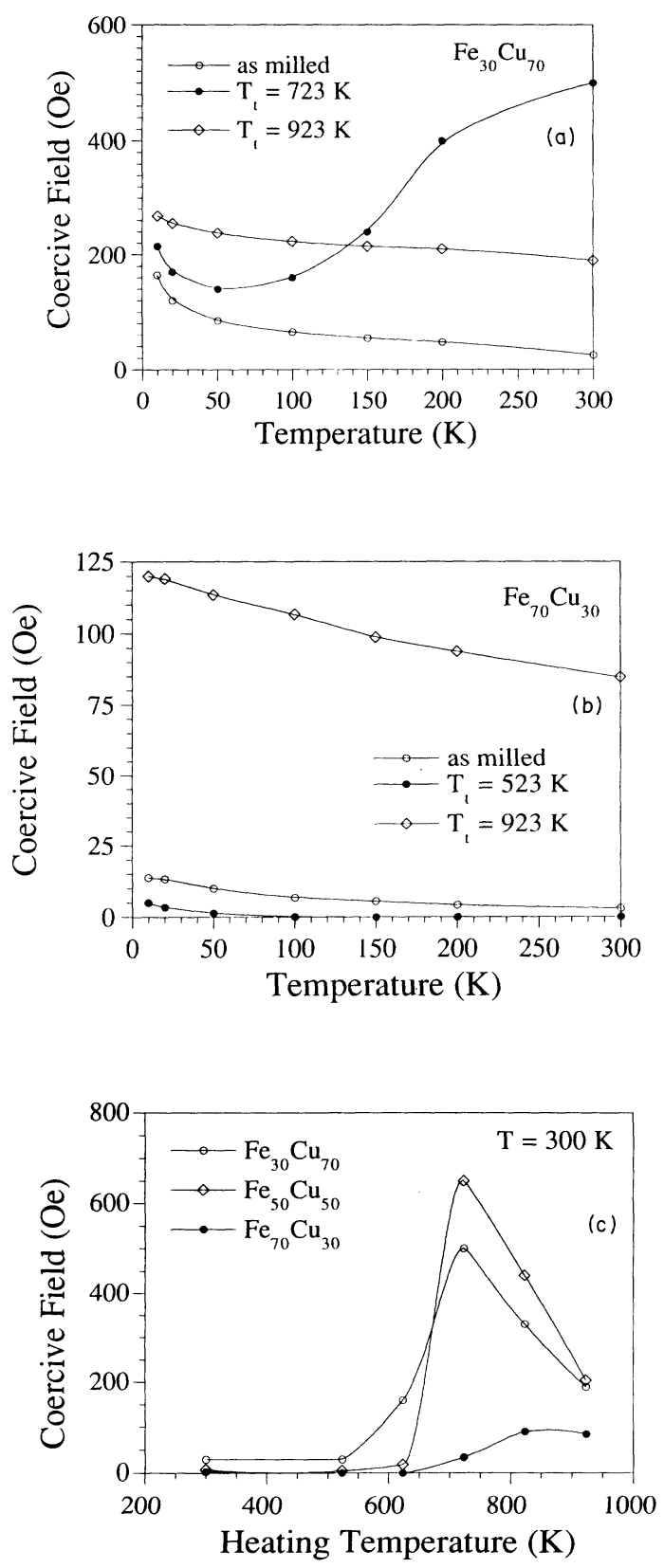

FIG. 3. Thermal dependence of the coercive field for samples (a) $x=0.3$, as-milled and treated at 723 and $923 \mathrm{~K}$, and (b) $x=0.7$, as-milled and treated at 523 and $923 \mathrm{~K}$. (c) Coercivity of the three alloys, measured at $300 \mathrm{~K}$, as a function of $T_{t}$.
$T_{t}=523 \mathrm{~K}$, according to Fig. 1, a decrease of the grain size must be disregarded and the superparamagnetic behavior can only be explained as a consequence of the exchange isolation of small ferromagnetic regions, originated by the transition to the paramagnetic state of their neighborhood volume. Therefore, the thermal dependence of the coercivity points out that the splitting of the Curie temperature of the sample components, well known from the dependence of saturation magnetization with temperature, ${ }^{7}$ takes place in a range of length shorter than the exchange correlation length. It is evident that the transformation exhibits characteristics of a spinodal transformation. Moreover, it is to be shown that the spinodal decomposition also accounts for the decrease observed in the magnetic moment.

The experiments indicate that the magnetic moment shows during decomposition a larger decrease for fcc than for bcc compounds (according to the results shown in Fig. 2, 38\% and $41 \%$ for samples with $x=0.3$ and 0.5 , respectively, against $9 \%$ for the sample with $x=0.7$ ). This fact can be understood by considering that the dependence on $x$ of the magnetic moment in the range $0.6<x<1$ is quite different for bcc and fcc structures. The magnetic moment of bcc alloys reaches a maximum for $x=1$ and linearly decreases as $x$ decreases up to 0.6 . For fcc alloys the magnetic moment increases linearly with $x$ up to $x=0.6$ and vanishes at $x=1$, thereby it should reach a maximum at $x_{m}$ placed somewhere between $x=0.6$ and 1 . If the alloy with initial $x=x_{0}$ decomposes via spinodal fluctuations the local $x$ fluctuates between $x_{1}$ and $x_{2}$ according to the amplitude of the modulation, with $x_{1}<x_{0}<x_{2}$. As the alloy tends to separate into the pure elements, $x_{1}$ and $x_{2}$ approach 0 and 1 , respectively, as the decomposition proceeds. When the structure is fcc the magnetic moment of both regions with $x_{1} \rightarrow 0$ and $x_{2} \rightarrow 1$ tends to zero; whereas in the case of bcc phases it only decreases in regions for which $x_{1} \rightarrow 0$. The large decrease of magnetic moment experimentally observed for the fcc sample, $40 \%$ in Fig. 2(b) implies that $x_{2}$ lies well above $x_{m}$, and therefore above $x=0.6$, in the region for which the spontaneous magnetic moment is negligible. The subsequent transition from the low magnetic moment fcc phase to the high magnetic moment bcc phase of those regions with high Fe content $(x>0.6)$ should produce an increase of the magnetization correlated to the growth of the bcc phase (Fig. 4). This magnetization increase has been observed for both samples with starting fcc phase [Figs. 2(a) and 2(b)]. As the grain size of the bcc phase increases (Fig. 1) the coercive field should decrease, which actually happens as shown in Fig. 3(c). According to this picture the initial decrease of the magnetic moment and its subsequent tendency to increase point out that during the spinodal decomposition a wide interval of the composition range for which the alloy cannot be produced in fcc structure stays in fcc phase. ${ }^{9}$

From the results obtained in the bcc sample, $x=0.7$ [Fig. 2(c)], it is also clear that during decomposition some regions in which $x_{1}$ is well below the production limit of bcc alloys, $x=0.6$, remains in the bcc phase. Furthermore, the magnetic moment decrease, of about $9 \%$ [Fig. 


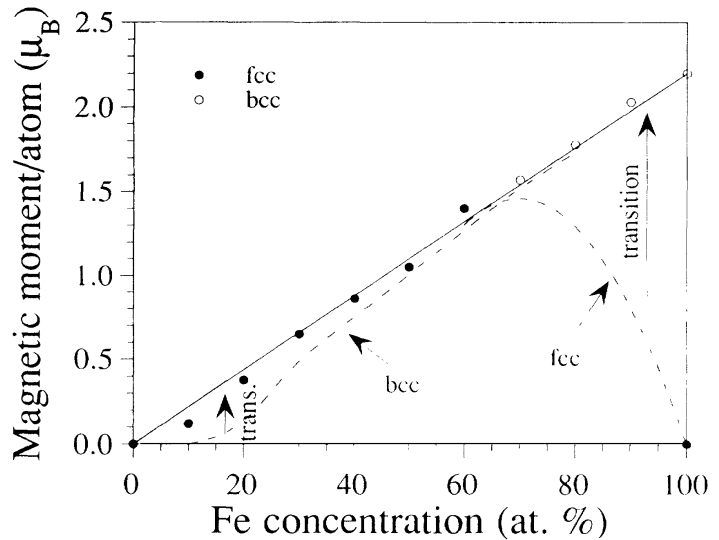

FIG. 4. The magnetic moment profile for bcc and fcc phases as a function of composition. Points indicate experimental results (10). The unknown magnetic moments have been tentatively drawn (dashed lines). Compositions that are as milled in the fcc branch undergo, during decomposition, a big magnetization decrease and also some recovery during the phase transition which takes place close to $x=1$. The magnetization of samples as milled with bcc structure decreases less during decomposition and also increases in a negligible amount during the phase transition which occurs at $x<0.5$.

2(c)], as well as the superparamagnetic transition indicates that the Fe magnetic moment in the bcc phase should vanish above $x=0$. The magnetization of the bcc sample does not show a tendency to increase during the bcc to fcc transition [Fig. 2(c)]. This is probably due to the low difference in magnetic moment corresponding to the bcc and fcc phases at the region in which the transition takes place $(x<0.5$, Fig. 4$)$.

It can be concluded that spinodal decomposition affects the magnetic moment up to $40 \%$. It produces splitting of the Curie temperature through the sample with a gradient of the order of $1000 \mathrm{~K}$ in a few nanometers. The splitting of critical temperatures in so wide range and in so short a distance scale allows the control of the magnetization process through changes in the temperature which generate variations in the exchange correlation length and consequently in the coercive force. Hence it is inferred that spinodal decomposition can be used to design and build up magnetic nanostructures. On the other hand it has been proven that magnetic measurements are ideal to inquire about details of the spinodal decomposition. In fact the decrease of magnetic moment has shown that ranges of compositions forbidden for $\mathrm{fcc}$ $(x>0.6)$ or bec $(x<0.6)$ structures when the alloy is produced by different methods become allowed for spinodal fluctuations.

This work has been supported by the Spanish CICYT through projects Mat. 92-0491 and Mat. 92-0405. A.H. is indebted to the BBV Foundation for support. We thank Dr. A. R. Yavari, Dr. A. L. Greer, and Dr. F. Gärtner for explaining to us the main features of spinodal decomposition.
${ }^{1}$ K. Uenishi, K. F. Kobayashi, S. Nasu, H. Hatano, K. N. Ishihara, and P. H. Shingu, Z. Metallkd. 83, 132 (1992).

${ }^{2}$ A. R. Yavari, P. J. Desre, and T. Benameur, Phys. Rev. Lett. 68, 2235 (1992).

${ }^{3}$ J. Eckert, J. C. Holzer, C. E. Krill, and W. L. Johnson, J. Appl. Phys. 73, 2794 (1993).

${ }^{4}$ C. L. Chien, S. H. Liou, D. Kofalt, W. Yu, T. Egami, and T. R. McGuire, Phys. Rev. B 33, 3247 (1986).

${ }^{5}$ A. Hernando, P. Crespo, J. M. Barandiaran, and A. Garcia Escorial, Phys. Rev. Lett. 70, 3521 (1993).

${ }^{6}$ A. R. Yavari, Phys. Rev. Lett. 70, 2235 (1993).

${ }^{7}$ P. Crespo, A. Hernando, A. R. Yavari, O. Drbohlav, A. Garcia Escorial, J. M. Barandiaran, and I. Orue, Phys. Rev. B 48, 7134 (1993).

${ }^{8}$ Jun-Ming Liu, R. Busch, F. Gartner, P. Haasen, Zhi-Guo Liu, and Zhuang-Chun Wu, Phys. Status Solidi A 138, 157 (1993).

${ }^{9}$ F. Gartner and A. L. Greer (private communications). For instance those regions of the fcc phase in which the local value of $x$ is above 0.6 would have less free energy in the bcc branch, and therefore they are subjected to a driving force oriented to produce phase transition. But due to the fcc environment the activation energy of the transition can be extremely high. During the time required by the kinetics to achieve the transition the spinodal mechanism continues acting and the $\mathrm{Fe}$ enrichment of these regions increases. The contribution of both tendencies explains that the limit $x=0.6$ can be overcome by many fcc local structures prior to undergo the phase transition. In fact a well-known method for production of fcc $\mathrm{Fe}$ is the epitaxy of a few layers on $\mathrm{Cu}$.

${ }^{10}$ E. Ma, M. Atzmon, and F. E. Pinkerton, J. Appl. Phys. 74, 955 (1993).

${ }^{11}$ TH. H. de Keijser, J. I. Langford, E. J. Mitteemeijer, and A. B. P. Vogels, J. Appl. Cryst. 15, 308 (1982).

${ }^{12}$ A. Slawska-Waniewska, M. Gutowski, H. Lachowicz, T. Kulik, and H. Matyja, Phys. Rev. B 46, 14594 (1992).

${ }^{13}$ A. Hernando and T. Kulik, Phys. Rev. B 49, 7064 (1994). 